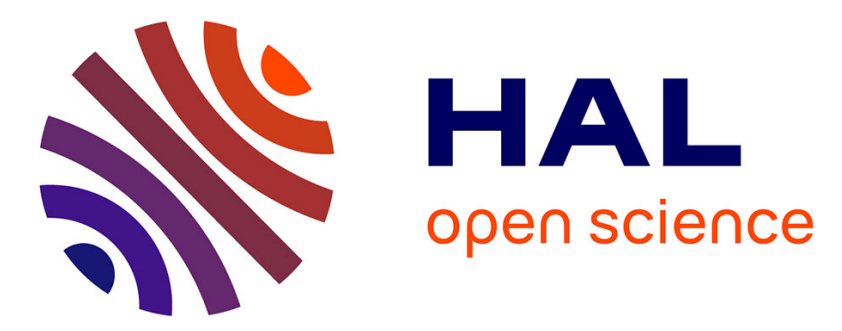

\title{
Les prémisses d'une restauration ? L'histoire enseignée saisie par le politique \\ Vincent Chambarlhac
}

\section{To cite this version:}

Vincent Chambarlhac. Les prémisses d'une restauration ? L'histoire enseignée saisie par le politique. Histoire@Politique: revue du Centre d'histoire de Sciences Po, 2012, 16 (1), pp.187 - 202. 10.3917/hp.016.0187 . hal-01623317

\section{HAL Id: hal-01623317 \\ https://u-bourgogne.hal.science/hal-01623317}

Submitted on 7 Feb 2018

HAL is a multi-disciplinary open access archive for the deposit and dissemination of scientific research documents, whether they are published or not. The documents may come from teaching and research institutions in France or abroad, or from public or private research centers.
L'archive ouverte pluridisciplinaire HAL, est destinée au dépôt et à la diffusion de documents scientifiques de niveau recherche, publiés ou non, émanant des établissements d'enseignement et de recherche français ou étrangers, des laboratoires publics ou privés. 


\section{LES PRÉMISSES D'UNE RESTAURATION ? L'HISTOIRE ENSEIGNÉE SAISIE PAR LE POLITIQUE}

Vincent Chambarlhac

Centre d'histoire de Sciences Po | «istoire@Politique »

2012/1 nº 16 | pages 187 à 202

Article disponible en ligne à l'adresse :

https://www.cairn.info/revue-histoire-politique-2012-1-page-187.htm

\section{Pour citer cet article :}

Vincent Chambarlhac, "Les prémisses d'une restauration ? L'histoire enseignée saisie par le politique », Histoire@Politique 2012/1 ( $\left.\mathrm{n}^{\circ} 16\right), \mathrm{p} .187-202$.

DOI 10.3917/hp.016.0187

Distribution électronique Cairn.info pour Centre d'histoire de Sciences Po.

(c) Centre d'histoire de Sciences Po. Tous droits réservés pour tous pays.

La reproduction ou représentation de cet article, notamment par photocopie, n'est autorisée que dans les limites des conditions générales d'utilisation du site ou, le cas échéant, des conditions générales de la licence souscrite par votre établissement. Toute autre reproduction ou représentation, en tout ou partie, sous quelque forme et de quelque manière que ce soit, est interdite sauf accord préalable et écrit de l'éditeur, en dehors des cas prévus par la législation en vigueur en France. Il est précisé que son stockage dans une base de données est également interdit. 
Vincent Chambarlhac, « Les prémisses d'une restauration ? L'histoire enseignée saisie par le politique », Histoire@Politique. Politique, culture, société, n 16, janvier-avril 2012, www.histoire-politique.fr

\title{
Les prémisses d'une restauration? L'histoire enseignée saisie par le politique
}

\author{
Vincent Chambarlhac
}

Le 27 août 2011, Le Figaro Magazine titre « Ce que nos enfants n’apprennent plus au collège », enquêtant sur un «scandale pédagogique et culturel ». Au coeur de la démonstration, l'affirmation implicite d'un abandon du roman national par l'Éducation nationale, ce même roman national que la Maison de l'histoire de France voulue par le président de la République devrait exposer ; ce malgré les polémiques, ce malgré un débat scientifique et syndical d'autant plus houleux qu'il implique la question des Archives de France'. Le roman national constitue aujourd'hui l'un des lieux où histoire et discours politiques se nouent d'autant plus que cette figure porte systématiquement, par la trame pédagogique qui la soutient, un horizon civique. La polémique n'est pas nouvelle, et l'on peut d'ailleurs lire les débats actuels comme les répliques du débat matriciel de 1979, où le politique saisissait l'histoire scolaire.

Le débat est connu, arpenté depuis peu par la sociologie du curriculum et l'intérêt porté à l'État éducateur en histoire de l'éducation² ${ }^{2}$ Le 20 octobre 1979, Alain Decaux publie un tonitruant appel dans Le Figaro, «On n'apprend plus l'histoire à vos enfants ! » ; la polémique s'ensuit, enfle, et aboutit à la restauration d'une histoire enseignée où s'impose le récit républicain, et où s'oublient systématiquement les tentatives de renouvellement pédagogique de l'enseignement de l'histoire entreprises dès avant $1968^{3}$. L'Association des professeurs d'histoire et de géographie (APHG) mobilise une grande part de la corporation dans ce débat et pèse sur cette restauration couronnée en 1985 dans les programmes. La controverse m'importe peu ici dans l'horizon des programmes; la manière dont le politique intervient, questionne l'épistémologie de la discipline enseignée et scientifique, forme la trame de cet article. Elle repose sur une hypothèse. François Cusset analyse la décennie 1980 comme un temps de restauration intellectuelle ${ }^{4}$. Le développement de

\footnotetext{
${ }^{1}$ Sur ce point, Vincent Duclert, J ean-Pierre Babelon, Ariane J ames-Sarrazin, Quel musée d'histoire pour la France?, Paris, Armand Colin, 2011.

2 Patricia Legris, " "On n'enseigne plus l'histoire à vos enfants!", Retour sur la polémique de l'enseignement de l'histoire de France au tournant des années 1970-1980 », dans J ulien Barroche et alii, Figures del'État éducateur, Paris, L'Harmattan, 2008.

3 On prendra comme signe ces deux publications de Suzanne Citron : un manifeste écrit en 1967 pour la société des professeurs d'histoire-géographie), également publié dans les Annales en 1968 (Suzanne Citron, «Pour l'aggiornamento de l'histoire-géographie», Bulletin de la société des professeurs d'histoire-géographie, octobre 1967. Publié dans la rubrique «Débats et combats » des Annales (janvier-février 1968) qui appelle à une réflexion et un renouvellement de l'histoire enseignée, et ce constat critique qui campe Suzanne Citron en contemptrice du récit national : Le mythe national, Paris, Éditions ouvrières, 1989.

4 François Cusset, La Décennie. Le grand cauchemar des années 1980, Paris, La Découverte, 2006.
} 
Vincent Chambarlhac, « Les prémisses d'une restauration ? L'histoire enseignée saisie par le politique », Histoire@Politique. Politique, culture, société, n 16, janvier-avril 2012, www.histoire-politique.fr

la polémique coïncide avec le centenaire des lois Ferry sur l’Éducation nationale, et s'il est une restauration dans le cadre de l'histoire enseignée ce ne peut être que celle du récit national, bientôt subsumée avec l'alternance politique, en récit républicain (1985), contre le « pédagogisme », le « didactisme » et l'importation dans le cadre de la discipline scolaire des problématiques d'une nouvelle histoire apte à émietter à l'infini tout récit. L'hypothèse posée est celle de la part du politique dans cette restauration ; du politique et non de l'État - lequel reste quasi en hors champ d'une analyse ancrée dans le vif de la polémique.

Pour Antoine Prost, la nation est un concept de politique intérieure; elle constitue dans l'ordre du discours politique un lieu où discriminer l'adversaire (politique), poser un bilan, dresser des perspectives. L'histoire enseignée par sa matrice républicaine représente un écran de premier ordre pour la projection de ces discours. Ainsi faut-il entendre le questionnement de la polémique en trois temps. Celui, d'abord, du temps bref d'une séquence politique où agissent des acteurs, se construit un sens donné à l'affrontement des idées. Celui de l'épistémologie ensuite, soit le questionnement au plus près des mots de ce qui se joue dans ce procès fait à l'histoire enseignée en 1979-1980: la restauration d'un édifice disciplinaire républicain supposé dégondé de sa vertu civique, rafraîchi alors à grand frais pour conjurer quoi, sinon le solde des années 1968 ? Le futur antérieur de la polémique, temps de l'historien et de l'après-coup, peut alors s'esquisser en une conclusion élargie, non pour cerner l'essence de la polémique mais en baliser son contexte. Un « moment » s'entrevoit là ; il n'est plus celui de Lavisse mais d'Alain Decaux, à lire Pierre Nora ${ }^{5}$. Un moment, soit une configuration politique et médiatique à explorer.

\section{Le tempo politique d’une polémique}

Dans l'attendu des programmes, la polémique ne se clôt définitivement qu'avec l'adoption des programmes Chevènement en 1985 - le grand colloque de Montpellier en 1984 représentant une inflexion significative. Au titre d'une analyse tramée par l'entrelacs de la polémique avec le politique, cette chronologie se resserre et ne s'étend in fine que de juillet 1979 à la fin de l'année 1980 quand l'approche de l'élection présidentielle clôt - momentanément - le débat. Cette courte séquence s'aborde par la chronique régulière d'Historiens et Géographes, revue de l'APHG, sur cette question, enrichie ensuite du contenu des revues.

Pour la littérature scientifique sur la question, l'article d'Alain Decaux lance la polémique. Celle-ci est puissamment relayée par une vague d'articles et de prises de position dans les journaux, dont l'APHG, qui sous la plume d'Hubert Tison, tisse une chronique serrée, alternant l'écho de la question dans les médias et le compte rendu de la présence de Clio à l'Assemblée nationale, au Sénat, dans Historiens et Géographes. À l'origine de la controverse, il y a le sort fait à l'histoire-géographie dans les projets de réforme du ministre René Haby largement investi depuis 1975 sur ce front de l'histoire, discipline enseignée qu'il s'agit d'insérer dans la modernité

\footnotetext{
5 Pierre Nora, «Alain Decaux raconte... Alain Decaux. Entretien avec Alain Decaux », Le Débat, mai 1984, n 30 , p. 45-80.
} 
Vincent Chambarlhac, « Les prémisses d'une restauration ? L'histoire enseignée saisie par le politique », Histoire@Politique. Politique, culture, société, n 16, janvier-avril 2012, www.histoire-politique.fr

giscardienne d'une "société libérale avancée », dont les programmes se discutent en 1978-19796 avec un nouveau ministre, Christian Beullac. La dilution de la discipline historique dans les blocs des « Humanités modernes » dans le secondaire, l'optionalité possible de la matière en Terminale, son insertion dans les activités d'éveil en primaire donnent aux contempteurs de l'action du ministère de l'Éducation nationale (MEN) le sentiment d'une disparition de l'histoire telle que traditionnellement enseignée. Si l'histoire n'est plus qu'une discipline d'éveil, sa finalité civique s'émousse évidemment aux yeux des contempteurs des réformes. L'article d'Alain Decaux restitue cette critique dès son chapeau introductif' :

«Pour les milliers de parents auxquels s'adresse ce cri d'alarme, c'est la stupeur:

l'école liquide l'histoire. Au nom de principes fumeux, une génération entière de petits

Français va-t-elle être coupée de ses racines profondes ? »

La polémique s'embrase rapidement ; elle a auparavant été préparée par une pétition à l'initiative de l'APHG dans Le Monde du 21 juillet 1979, réunissant 401 signatures. L'APHG relaie ces signatures par la production d'un imposant courrier des lecteurs alternant prise de position collective (ainsi de l'arc syndical du SNALC - Syndicat national des lycées et collèges - au SNES - Syndicat national des enseignants du second degré - , via le SGEN-CFDT - Syndicat général de l’Education nationale - , mais aussi de l'UER - Unité d'enseignement et de recherche - d'histoire de Lille III) et individuelle (enseignants, conservateurs, bibliothécaires, députés, chercheurs,

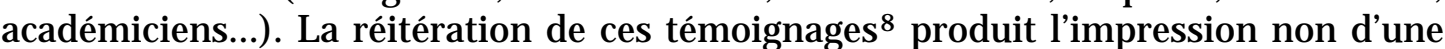
profession mobilisée mais plutôt d'un pays dressé contre son gouvernement. Ainsi s'agit-il moins d'aborder la question du corporatisme de l'APHG mais bien davantage l'effet de sens politique de cette coupure où s'exhibe un maillage serré du territoire par les témoignages. En matière d'histoire enseignée, le pays réel s'opposerait au pays légal.

On reconnaît là un dispositif efficace de structuration de la mobilisation politique souvent employé, sur le siècle, par la droite. Elle s'empare d'une polémique qui paraît, dans la succession des prises de position, une affaire d'abord interne à la majorité politique. En septembre-octobre 1979, Michel Debré dresse par deux fois le procès des projets du MEN qui sacrifie l'enseignement de l'histoire et de la géographie : dans un entretien accordé aux Nouvelles littéraires (13 septembre 1979), puis dans La Lettre de Michel Debré $\left(n^{\circ} 22-23\right)$; il le réitère dans Le Figaro du 10 octobre 1979. Auparavant, comme Alain Devaquet notamment, il avait affirmé son soutien à la cause de l'APHG. La chronologie fait sens, et l'on perçoit l'appropriation du débat initié en juillet 1979 par le RPR contre l'UDF. Le contexte politique d'une majorité éclatée s'additionne de la thématique de l'Appel de Cochin lancé le 6 décembre 1978 pour les premières élections au suffrage universel au Parlement européen. L'UDF est là le « parti de l'étranger » et l'on mesure mieux pourquoi dans le sillage de cette ligne politique les cadres du RPR s'engagent sur la question de

\footnotetext{
6 Pour un panorama plus global du projet, du rôle de René Haby, cf. Patricia Legris, op. cit., note 1.

7 Alain Decaux, « On n’apprend plus l’histoire à vos enfants, Le Figaro, 20/10/1979.

8 Chacun des numéros d'Historiens et Géographes pour le second semestre 1979 comporte la reproduction des témoignages de soutiens de lecteurs et/ ou d'institutions.
} 
Vincent Chambarlhac, « Les prémisses d'une restauration ? L'histoire enseignée saisie par le politique », Histoire@Politique. Politique, culture, société, $n^{\circ} 16$, janvier-avril 2012, www.histoire-politique.fr

l'histoire enseignée9. Michel Debré donc, mais aussi Jean-Pierre Bachmann (délégation départementale du RPR pour l'éducation à Paris) dans La Lettre de la Nation (03/ 10/ 1979), Sylvie Dreyfus (Le Figaro, 10/11/ 1979). De proche en proche, le dispositif éditorial de la mouvance du RPR se mobilise : Le Figaro et son magazine, évidemment, mais aussi Le Point, Valeurs Actuelles sous la plume de Frédéric Valloire... Au Parlement, les premières chroniques d'Hubert Tison mentionnent également la rapide appropriation par les parlementaires RPR des thématiques de la polémique: Michel Debré, Gabriel Kaspereit, Alain Devaquet ${ }^{10} \ldots$ Enfin, en collaboration avec Historia et Historiens et Géographes, Michel Debré organise un colloque sur «L'enseignement de l'histoire dans la jeunesse » le 25 juin 1980, dont une part des actes sera publiée ${ }^{11}$.

Cette première configuration de la polémique par la médiation politique du RPR se structure sur plusieurs lignes. Au plus près du politique, l'implicite figure de l'UDF comme «parti de l'étranger» nourrit une lecture complotiste du sort fait à l'enseignement de l'histoire dans son rapport au national : " Ceux qui aspirent à ce qu'il n'y ait plus de France se sont emparés de l'Histoire» déclare Michel Debré (Historiens et Géographes, $n^{\circ} 276$, décembre 1979). Cochin résonne là évidemment, mais la figure discursive trouve rapidement des échos à l'extrême droite. Par l'éclat de la prise de position d'Alain Decaux la polémique enfle, Clio entre au Parlement. Derechef, la politisation de la polémique s'étoffe d'autres lignes que celle issue du RPR. L'APHG, tout à son travail de défense de la profession, rencontrait le PS dès mai 197912. Celui-ci a peu réfléchi sur la question de l'histoire, sa commission éducative se tournant davantage sur la question de l'enseignement professionnel. La question du sort fait à l'histoire-géographie s'appréhende pour le PS comme un trait classique du capitalisme qui «spécule sur l'amnésie et l'inculture historique »; lecture qui lui permet la promesse de souhaiter « ressusciter la mémoire et redonner un sens à l’histoire de France » (Historiens et Géographes, n² 276, décembre 1979)».

9 Ce lien des problématiques de l'Appel de Cochin à la défense de l'histoire existe très tôt chez Michel Debré qui dès le 17 février 1979 propose cette question écrite au ministre de l’Éducation nationale : M. le ministre « n'estime pas que la part faite à l'étude de l'histoire de France et la géographie de la France est désormais trop faible dans nos programmes scolaires; qu'en effet il est frappant de constater que les principales connaissances qu'ont les jeunes du passé ou de la configuration de leur pays vient non de leur instruction scolaire mais des images et des films de la télévision ; qu'au surplus la préparation des Français à leurs responsabilités exige une connaissance profonde de leur histoire et de leur géographies nationales et qu'il paraît préoccupant de voir des soucis d'ordre régional ou supranational prendre le pas sur l'enseignement fondamental pour l'avenir de la nation et de la République ». (Historiens et Géographes, $n^{\circ} 273$, mai-juin 1979). Au plus fort de la polémique, la dénonciation s'affine et se radicalise.

10 Cf. les chroniques d’Historiens et Géographes: n²73 (mai-juin 1976), nº 276 (décembre 1979) notamment.

11 Patrick Garcia, «Valéry Giscard d'Estaing, la modernité, la politique et l’histoire », dans Claire Andrieu, Marie-Claire Lavabre, Danielle Tartakowsky (dir.), Politiques du passé. Usages politique du passé dans la France contemporaine, Aix-en-Provence, Presses universitaires de Provence, 2006, p. 119-132.

12 Rencontre de: A. Bolliet, R. Fossier, H. Tison, M. Pécheux pour l'APHG avec Louis Mexandeau (délégué national du PS à l'Éducation nationale), J ean-Louis Piednoir (secrétaire de la Commission éducative nationale du PS), François Aron (membre de cette commission) le 16 mai 1979. Historiens et Géographes, $n^{\circ} 275$, septembre-octobre 1979. 
Vincent Chambarlhac, « Les prémisses d'une restauration ? L'histoire enseignée saisie par le politique », Histoire@Politique. Politique, culture, société, n 16, janvier-avril 2012, www.histoire-politique.fr

En soi donc, une forme d’acquiescement à la nécessité de réinscrire le récit national au coeur des programmes d'histoire. Le PCF lit lui dans ce sort fait à l'enseignement de l'histoire la volonté gouvernementale d'effacer l'expérience des luttes par l'escamotage du récit national. Dans son discours affleure la part prépondérante de l'histoire leçon politique pour qui use du matérialisme historique pour la lire et l'enseigner. La dénonciation du projet s'inscrit dans la logique des pistes proposées contre la réforme Haby depuis 1975, celle d'un réquisitoire contre une histoire dénaturée qu'énonce Claude Mazauric :

" Mais qui ne voit que sous couvert de "changement" dans le contexte de la société et de l'école actuelles, avec ce Pouvoir ce que l'on entreprend n'est pas de favoriser un meilleur enseignement de l'histoire, plus scientifique, plus libérateur, plus transformateur du réel présent, mais de distiller dans un ensemble sans statut scientifique reconnu des "savoirs" et des "vocabulaires" mystificateurs, au terme d'une démarche essentiellement téléologique, sans même l'inventaire ni la mise en forme préalable des objets de connaissance, de concepts et des méthodes qui s'imposent à tout enseignement objectif ${ }^{13}$. »

Après les communiqués, les articles de presse et les déclarations, le second temps de l'action politique est parlementaire. À la manouvre, le RPR propose une loi d'orientation sur l'enseignement de l'histoire, jamais discutée mais publiée in extenso par Historiens et Géographes dans leur numéro de novembre 1980. L'artisan de la constitution qu'est Michel Debré défend face au ministre Christian Beullac à l'Assemblée nationale l'idée que les manuels - supports des nouveaux programmes dans le champ médiatique - « s'efforcent de troubler les esprits par une présentation systématique des faits et des hommes propres à dégoûter les jeunes de leur pays », arguant qu'en conséquence « la Constitution est violée [puisque] le Parlement aurait dû en effet statuer, puisque l'enseignement de l'histoire est un principe général de l'enseignement qui relève de la loi ${ }^{14} \gg$. Si le ministre botte en touche, l'évidence politique du débat et la volonté de l'inscrire au coeur du Parlement ne fait aucun doute. À l'instar du RPR, Louis Mexandeau pour le PS multiplie les questions écrites au MEN s'attaquant également au contenu des manuels, supposés refléter les contenus des cours. L'horizon de l'élection présidentielle étire ces débats qui, peu à peu, s'enlisent.

Que conclure de cette première focale posée sur le temps court de la polémique? L'idée d'abord - souvent minorée - d'une part essentielle du politique dans la médiatisation de la cause défendue par l'APHG. Le RPR se montre l'artisan du débat, par le truchement d'un Michel Debré, omniprésent en coulisses semble-t-il, apte à médiatiser à l'Assemblée les débats. Michel Debré joue là également une carte personnelle: il est alors candidat à l'élection présidentielle de 1981, bien que marginal au sein du RPR. Son engagement pour la cause de l'histoire scolaire peut également se lire comme un choix opportuniste apte à favoriser sa plus grande visibilité dans le débat politique. La politisation de la polémique procède de ces ressorts. Son succès médiatique enjoint au PS de s'en emparer. La question de

${ }^{13}$ Claude Mazauric, « L'histoire dénaturée », L'École et la Nation, n² 252, juin-juillet 1975, p. 40-43.

${ }^{14}$ Assemblée nationale, séance du vendredi 30 mai 1980. 
Vincent Chambarlhac, « Les prémisses d'une restauration ? L'histoire enseignée saisie par le politique », Histoire@Politique. Politique, culture, société, n 16, janvier-avril 2012, www.histoire-politique.fr

l'histoire enseignée s'inscrit à l'agenda des colloques socialistes (Historiens et Géographes, $n^{\circ} 275$, septembre 1979) bien que le PS n'ait pas avant spécifiquement consacré une part de sa réflexion à la question historique ${ }^{15}$. Pour J ean Leduc et Patrick Garcia, l’année 1980 marquerait une forme d'union sacrée politique contre les projets de réforme du gouvernement ${ }^{16}$. La formule ne fonctionne évidemment qu'en regard d'une histoire de l'enseignement ; elle convient à la manière dont l'APHG pose le débat, arguant que cette question est une « affaire d'État ${ }^{17}$ », martelant l'idée que cette « campagne fut menée en toute indépendance [par l'APHG] » afin d'éviter l'épithète de réactionnaire, ou conservatrice opposé par d'aucuns ${ }^{18}$. Dans cette manière de camper la polémique, l'APHG use implicitement de la figure d'un front unique à vocation défensive, où par l'argument de «l'affaire d'État » s'évacueraient les connotations propres au champ politique qu'impliquent ces épithètes. Ils procèdent pourtant de la politisation même de la polémique, et invitent à juger dans les termes des enjeux politiques véhiculés par l'enseignement de l'histoire le procès rétrospectif qui lui est fait.

\section{Le temps rétrospectif du procès}

L'hypothèse d'une restauration de l'enseignement du récit national implique la parenthèse que serait l'ordre contemporain de l'histoire enseignée. Pour J ean Peyrot, cette parenthèse qu'il s'agit de refermer est le « temps des calamités », dont il date les prémisses autour de $1961^{19}$. La titulature apocalyptique cerne le « moment 68 » dont on sent bien qu'il s'agit d'entreprendre dans l'horizon de l'histoire enseignée le procès. Structurellement, la polémique réarticule une doxa pérenne dans les discours sur l'École entre conservateurs et réformistes ${ }^{20}$ sur le bilan de ce «moment 68 » ; l'APHG et la majeure partie des polémistes appartiennent au camp conservateur, s'engageant pour une restauration des savoirs enseignés, plaçant au banc des accusés, les réformistes, tout entiers confondus dans la dénonciation des réformes Haby. Louis François, doyen de l'Inspection générale d’histoire géographie, révèle au débat organisé à la Fnac (Forum des Halles, 6-7 mars 1980) «les responsabilités de René Haby dès 1962 dans l'amoindrissement de nos disciplines », comme le note la chronique d’Historiens et Géographes ( ${ }^{\circ}$ 278, mai 1980). 1962, soit l'entrée de René Haby au MEN comme directeur de la pédagogie. On ne saurait mieux restreindre une

\footnotetext{
${ }^{15}$ Cf. la publication en 1978 par Louis Mexandeau et Roger Quillot de Libérer l'école : plan socialiste pour l'Éducation nationale (Paris, Flammarion, 1978). La question de l'histoire, telle que posée par la polémique, sera intégrée au colloque de Grenoble au volet consacré à la formation des maîtres, ce qui implique une saisie en oblique des problématiques de la polémique.

16 Patrick Garcia, Jean Leduc, «Enseignement de l'histoire en France», dans Historiographies. Concepts et débats, tome I, Paris, Folio histoire, 2010.

${ }_{17}$ J ean Peyrot, « Garder le cap », Historiens et Géographes, n 278, mai 1980, p. 545.

18 Hubert Tison, «L'histoire et la géographie dans les médias », Historiens et Géographes, n 277 , février-mars 1980, p. 373.

19 Jean Peyrot, "Rétrospective pour une prospective», Historiens et Géographes, n²79, juinjuillet 1980, p. 717-723.

20 Marie-Anne Paveau, «Formes et fonctions de la doxa dans les discours sur l'École», Mots, décembre 1999, n 61, p. 9-27.
} 
Vincent Chambarlhac, « Les prémisses d'une restauration ? L'histoire enseignée saisie par le politique », Histoire@Politique. Politique, culture, société, n 16, janvier-avril 2012, www.histoire-politique.fr

séquence chronologique complexe à un nom... La restitution de cette doxa au gré des colloques et des prises de position repère les points forts de ce qui semble faire consensus pour le front constitué par l'APHG ; elle désigne politiquement en creux des adversaires par des termes clés du lexique de l’APHG: Risorgimento, aggiornamento.

Les premières chroniques de l'APHG dénoncent « un enseignement amnésique, sans racines et sans espaces » (Historiens et Géographes, $n^{\circ} 273$, mai-juin 1979, p. 531). Rapidement la topique du procès s'étend pour Le Figaro sous la plume de Lucien Crenne à une « crise globale de l'enseignement français. Au-delà de l'histoire, c'est la transmission même de la culture qui est menacée» (24/11/1979). La pluridisciplinarité constitue la cible de ces discours qui s'épaulent l'un l'autre, s'adossent aux contributions de revues plus spécifiques au champ historique comme H. Histoire, Historia, L'Histoire. Mesurée à l'aune des programmes et des conditions d'exercice du métier par l'APHG, cette dénonciation embrasse des formes plus caricaturales dans l'enceinte parlementaire, ainsi de cette question écrite du sénateur Guy Robert (UCDP) le 26 octobre 1979, qui comprend cette incise, destinée à convaincre :

« De grâce, que la génération de demain ne soit pas plus au fait des aventures de Goldorak et de Superman que de ce que furent la vie, les combats de Vercingétorix, de Saint Louis ou de J eanne d'Arc. »

L'APHG reconnaît qu'il « existe un malentendu entre l'APHG qui s'efforce de montrer son souci de dépoussiérer l'événement de nos disciplines et les députés et les sénateurs qui, sur notre suggestion, interviennent pour les défendre, ce qui est souhaitable, mais semblent parfois réclamer le retour à un enseignement traditionnel » (Historiens et Géographes, $n^{\circ} 276$, décembre 1979). Pour autant, la topique du front comme l'appui inconditionnel de l'association apporté à Alain Decaux marque politiquement ce combat dans l'horizon des discours de « retour au village » analysé par Marcel Detienne ${ }^{21}$. L'insistance posé dans le discours politique sur le constat de crise de civilisation accompagne ces discours ; l'identitaire rassure, à l'histoire enseignée de l'établir durablement pour une jeunesse réputée d'autant plus indocile que l'Ecole s'est massifiée. Dans cette logique, la référence de J ean Peyrot « au temps des calamités » vise à restaurer un ordre scolaire dont le colloque organisé par Historia le 4 mars 1980 fournit les attendus. Pour l'APHG, « enseignants et spécialistes y sonnent le tocsin », et il revient à Pierre Goubert d'asséner, avec force applaudissements, que la situation est de fait le résultat « d'une offensive d'obscurantisme méchant qui se dissimule sous le charabia imité des sociologues et pédagogues américains démodés depuis quinze ans ». J ean-Pierre Chevènement reprend l'argument antiaméricaniste ${ }^{22}$; la salle semble faire consensus malgré les divergences politiques. La charge de Pierre Goubert s'achève sur la

\footnotetext{
${ }^{21}$ Marcel Detienne, Comment être autochtone. Du pur athénien au français raciné, Paris, Seuil, 2003.

22 Ré-emploi d'autant plus facilité qu'il ne cesse dans le parti socialiste de désigner la « deuxième gauche» comme une gauche américaine. Or la deuxième gauche d'alors - proche de la mouvance cédétiste - compte nombre de pédagogues. D'une pierre deux coups donc, à l'heure où Michel Rocard s'est affirmé candidat, quand François Mitterrand ne s'est pas encore décidé.
} 
Vincent Chambarlhac, « Les prémisses d'une restauration ? L'histoire enseignée saisie par le politique », Histoire@Politique. Politique, culture, société, n 16, janvier-avril 2012, www.histoire-politique.fr

« nécessité de faire passer l'air pur dans les couloirs des instituts pédagogiques, des ministères et renvoyer les pédagogues à l'enseignement » (Historiens et Géographes , $\mathrm{n}^{\circ}$ 278, mai 1980). Sur le fond, les participants au colloque d'Historia, tout en souhaitant se garder d'une histoire chauvine, articulent l'ensemble de leur projet sur une réintroduction poussée de la chronologie et du national. L'éviction des pédagogues, « renvoyés à l'enseignement », nécessite également que se réarticule le lien entre praticiens de l'enseignement et producteurs de l'histoire. L'horizon (onirique) du projet tient tout entier dans la restauration mythique de l'édifice scolaire de Jules Ferry, quand l'histoire devient enseignement et discipline scientifique ; le panorama ne peut alors se dire qu'au prix de la mise en accusation des pédagogues. Logiquement, le colloque d’Historia constitue l'acmé de la polémique, ramenant à l'épure conservatrice l'idéal de restauration donné comme réforme nécessaire.

Le vocabulaire a sa part dans la mise en scène de la polémique. Historiens et Géographes, par Hubert Tison, qualifie de Risorgimento le front polémique dont l'APHG serait la cheville ouvrière. L'emprunt à l'histoire italienne du XIX ${ }^{\mathrm{e}}$ siècle peut se lire comme un clin d'œil à Alain Decaux - ardent défenseur de ce siècle. Il dit surtout un positionnement dans l'espace politique de la nation cadre unitaire contre l'émiettement régional. Dans le lexique des usages politiques de l'histoire après mai 1968, on entendra là la défense du récit national contre l'histoire d'en bas, populaire. Le discours tenu heurte alors frontalement des pratiques de l'histoire tenues par des collectifs, certes minoritaires, mais qui pesèrent sur la définition de l'histoire, du rôle de sa pratique. Au plus près de la charge de Pierre Goubert, les propositions de J ean Chesneaux (Du passé faisons table rase? À propos de l'histoire et des historiens, Paris, Maspéro, 1976) et le travail des Cahiers du Forum-histoire qui viennent de fournir leur dernière livraison. Collectif de chercheurs et de professeurs, ils s'efforçaient de penser et d'enseigner une autre histoire, distincte des canons de la IIIe République et du récit national. Dominique Dhombres résumait ainsi leurs propositions :

« L'histoire scolaire d'aujourd'hui, celle des programmes officiels et des manuels a été construite sous la IIIeRépublique, ont expliqué des enseignants. Cette histoire gouvernementale et institutionnelle, constituée à partir d'archives officielles et de documents diplomatiques favorise dans le tissu historique tout ce qui concerne le pouvoir central et les institutions, et les rapports d'État à État. Les jeunes réfutent cette histoire. Ils lui opposent l'indifférence ennuyée, le chahut ou l'acceptation résignée quant elle devient objet d'examen ou de concours. »

« Le ton du forum était donné. Il s'agissait de faire parler ceux qu'écrasent aujourd'hui les discours érudits des professionnels de l'histoire, les classes populaires, les femmes, les minorités de l'Hexagone. (...).

« La commission sur « la crise de l'histoire à l'école » a élaboré une « déclaration des droits du professeur d'histoire et de géographie qui laisserait à celui-ci la possibilité de faire travailler des élèves sur «l'histoire immédiate » de leur ville ou de leur région 
Vincent Chambarlhac, « Les prémisses d'une restauration ? L'histoire enseignée saisie par le politique », Histoire@Politique. Politique, culture, société, n 16, janvier-avril 2012, www.histoire-politique.fr

afin de leur montrer que l'histoire n'est pas une abstraction lointaine, mais qu'ils en sont eux-mêmes les acteurs ${ }^{23}$. »

Les buts de Forum-histoire, leur intérêt pour l'histoire locale, leur défiance pour les « maîtres à penser », comme leur appétence pour le contre-manuel trahissent une filiation directe avec Mai 1968. Le collectif s'est créé en 1975 à Paris VII dans la filiation du reflux gauchiste après 1974, il souhaite mettre l'accent sur les relations « explicitement politiques entre les priorités du présent et l'étude du passé24», Enseignants d'histoire, historiens - professionnels ou amateurs - s'unissent dans ce collectif pour promouvoir la pratique de l'histoire d'en bas contre le récit national, unificateur. La pratique de l'histoire par le collectif s'inspire pour partie de l'history from below théorisée outre-Manche par E.P. Thompson ${ }^{25}$, et réfute par son appétence pour la révolte, le discours d'ordre, construit autour de l'État et de la Nation, qu'est le roman national. Sour en revue des Cahiers du Forum-histoire, dont il partage une égale matrice gauchiste, Le Peuple français récuse de même le contenu du colloque d'Historia, les prises de positions favorables à la cause de l'APHG.

«La rédaction du Peuple français voit dans ces déclarations une véritable philosophie antipopulaire de l'Histoire : ce serait l'individu et non le peuple qui ferait l'histoire...» L'éditorial s'attaque aux mandarins des Annales et de l'École pratique des hautes études «qui veulent faire pièce à toute tentative d'écrire une véritable histoire populaire... (...) Tous ces mandarins ont donc délaissé l'histoire des élites et abordé l'histoire des "phénomènes de longue durée" sous prétexte de remettre l'histoire sur ses pieds, ils développent leurs études sur des échelles chronologiques vastes. Certes ils réintègrent les gueux, les marginaux, les paysans... mais c'est pour mieux présenter l'histoire quasi immobile de l'homme. Le peuple ne serait pas acteur de l'histoire mais la subirait ${ }^{26} \gg$.

Laissons de côté l'épistémologie sous-jacente du propos pour revenir au qualificatif de Risorgimento. Le choix du terme par Hubert Tison doit se lire politiquement. Il atteste d'une part la volonté du récit national contre l'éclatement d'une histoire construite à partir du local puisque celle-ci dans la rhétorique du « moment 68 » désajuste le lien des petites patries à la patrie, se donnant là comme une charge critique de la fonction civique du professeur d'histoire, rabattu sur la figure mandarinale du maître à penser. Il implique d'autre part la volonté éminemment restauratrice du combat mené par l'APHG, si l'on admet qu'en l'espèce la défense de la corporation et de l'édifice à laquelle elle s'adosse vaut médiation politique. Le discours du maître doit s'entendre pour que l'histoire ne soit pas populaire au sens post-soixante-huitard d'une histoire qui déconstruirait les catégories du national. Ramenée sur le plan pédagogique, la proposition implique la dénonciation du « tout pédagogique », soit notamment de l'Institut national de recherche pédagogique -

23 Dominique Dhombres, «Des professeurs veulent enseigner une autre histoire», Le Monde, 29 mai 1975

24 J ean Chesneaux, L'engagement des intellectuels (1944-2004). Itinéraire d'un historien franc-tireur, Cahors, Privat, 2004.

25 Sur cette position de l'history from below, cf. Arnaud Mattelard et Éric Neveu, Introduction aux Cultural Studies, Paris, La Découverte, 2003

26 « Liquidation ou commercialisation de l'histoire », Le Peuple français, avril-mai 1980. 
Vincent Chambarlhac, « Les prémisses d'une restauration ? L'histoire enseignée saisie par le politique », Histoire@Politique. Politique, culture, société, n 16, janvier-avril 2012, www.histoire-politique.fr

INRP - fraîchement réorganisé (1976), identifié alors - à tort ou à raison - à la modernisation giscardienne. La reprise de la thématique de l'aggiornamento évite alors l'accusation de conservatisme ou de réaction - pourtant tellement palpable qu’à longueur de chroniques l'APHG ne cesse de s'en défendre, au risque du lapsus.

Au ras du langage conciliaire, aggiornamento semble le terme adéquat, impliquant l'ouverture sur la modernité de Vatican II ; la polémique ne réclame rien d'autre d'ailleurs qu'une mise à jour. Dans l'épaisseur historique des tentatives de moderniser l'enseignement de l'histoire au sein du MEN, l'emploi d'aggiornamento vaut détournement d'autant plus signifiant qu'il en inverse les polarités modernisatrices. En octobre 1967, Suzanne Citron publie « Pour l'aggiornamento de l'histoire-géographie » dans le Bulletin de la société des professeurs d'histoire et de géographie ; par l'entremise de Fernand Braudel, l'article est repris dans les Annales en 1968 sous le même titre dans la rubrique «Débats et combats ». Notons que l'aggiornamento ne se donne ni comme un manifeste, ni comme une rupture, mais s'inscrit dans le cercle des propositions recevables alors par l'institution; l'organisation du colloque d'Amiens (mars 1968) par l'inspection générale où ces problématiques sont largement discutées semble l'établir, comme la tenue cette même année du colloque de Sèvres et d'une journée d'étude consacrée à l'enseignement de l'histoire. Le document, et la pratique pédagogique qu'il enjoint, forment le point de jonction entre l'histoire universitaire et sa pratique scolaire; en cela, les propositions de Suzanne Citron s'inscrivent dans les renouvellements en cours de l'histoire scolaire par la notion d'éveil et l'irruption de dossier documentaire dans les manuels. La publication des nouveaux programmes de collège en 1969 rompt brutalement cette logique, supposant contre les aspirations de Mai 68 une reprise en main del'Inspection générale et un retour de la hiérarchie ${ }^{27}$. Suzanne Citron continue alors son combat aux marges de l'institution, s'appuyant sur une structure Enseignant 70 - puis par l'animation du «groupe de Villetaneuse » actif dans le cadre de la formation continue des enseignants; son militantisme la porte sur la volonté de « déscolariser la société ». Elle côtoie alors Forum-histoire, entre au PS («Deuxième gauche ») dans la mouvance rocardienne. Militante infatigable d'une rénovation de l'enseignement, elle s'oppose systématiquement à un enseignement de l'histoire dont la conception dérive en droite ligne de la III République, s'opposant ainsi au roman national ${ }^{28}$. Dans ce parcours, le texte de 1967 a son importance et peut d'autant moins être ignoré de l'APHG qu'il fut publié dans son bulletin. La dénonciation de l'action de l'APHG par Suzanne Citron aux côtés de Daniel Hémery dans Le Monde n'en a que plus de poids :

«Va-t-on revenir à l'ambiguïté de la défense de l'enseignement de l'histoire, qui remonte au moins à la fondation de la Société des Professeurs d'histoire (1910) et qui mêle étroitement - et inconsciemment - défense de statut et identitaire (défense du moi enseignant de l'histoire) à une conception magico-mystique de l'histoire, cette

\footnotetext{
27 Suzanne Citron, «Un parcours singulier dans la fabrique de l'histoire », dans Laurence de Cock, Emmanuelle Picard, La fabrique scolaire del'histoire, Marseille, Agone, 2009, p. XVII-XXI.

${ }^{28}$ Suzanne Citron, Mes lignes de démarcation. Croyances, utopies, engagements, Paris, Syllepse, 2003.
} 
Vincent Chambarlhac, « Les prémisses d'une restauration ? L'histoire enseignée saisie par le politique », Histoire@Politique. Politique, culture, société, n 16, janvier-avril 2012, www.histoire-politique.fr

dernière excluant toute distanciation critique vis-à-vis de la fresque historique

traditionnelle des programmes et des manuels ${ }^{29}$ ? »

L'emploi du terme d'aggiornamento par l'APHG n'est pas neutre alors ; il signifie la fin d'un moment ouvert dans les années 1960, celui de la rénovation pédagogique, au profit d'une restauration de l'équilibre antécédent. Le retournement est d'autant plus violent qu'à douze ans d'intervalle il s'énonce sous le même mot-valise. Marquée de l'idéologie de retour au village, la polémique participe pleinement d'une phase de restauration intellectuelle, dont elle constituerait une forme de prémisses. La trame du procès rétrospectif fait à l'enseignement de l'histoire congédie le « moment 68 », ses ombres gauchistes et ses explorations pédagogiques. Le colloque organisé par Historia forme l'acmé de ce mouvement qui, in fine, pourrait s'apparenter à un jeu à somme nulle du fait de l'immobilisme du MEN, du fait aussi du mouvement de reflux d'une part des soutiens de l'APHG comme semble le souligner la chronique qui va s'amenuisant au fil de 1980. Il n'est qu'une lecture inscrite dans la temporalité des programmes pour tisser une véritable continuité entre les textes de 1979 et les programmes Chevènement de 1985. Au titre du politique, les rythmes sont plus courts. C'est donc au présent de ce moment qu'il s'agit d'en discuter la teneur politique.

\section{Le présent d'un moment}

L'hypothèse d'une restauration intellectuelle, dont la polémique constituerait les prémisses, suppose de sonder ce «moment» singulier du rapport de la société française à l'histoire enseignée. Pierre Nora interviewant Alain Decaux décèle un « moment Decaux ${ }^{30}$ ». L'expression vaut épistémologiquement que l'on s'y attarde. Son surgissement éphémère coïncide avec l'édition du premier volume des Lieux de mémoire qui consacre historiographiquement un «moment Lavisse », et compte nombre d'articles historicisant l'École de Jules Ferry. La symétrie dit ici le rapport de Pierre Nora à l'histoire, des questions du présent au passé31. Revenant sur les Lieux de mémoire, il en saisit ainsi la trajectoire en 1995 :

"L' "Histoire de France" est un genre que Les Lieux de mémoire ont voulu à la fois subvertir et illustrer. Subvertir en faisant du genre et de cette histoire un objet d'histoire, et donc en privilégiant la dimension historiographique. Subvertir aussi en substituant à l'affirmation d'une unité organique l'analyse de cette construction, réelle ou imaginaire, de ses instruments et de ses moyens. Subvertir, enfin, en la laïcisant et en la démocratisant. Mais aussi, par l'ampleur des proportions et des qualités du traitement, s'inscrire dans cette grande tradition. Toutes les grandes histoires de France, d'Étienne Pasquier, au XVIe siècle, à Michelet, et de Michelet, à Lavisse et à Braudel, commencent où s'achèvent sur une déclaration d'amour à la France, une

\footnotetext{
29 Suzanne Citron, Daniel Hemery, Le Monde, 17 mai 1980.

30 Pierre Nora, « Alain Decaux raconte...», op. cit.

31 François Dosse, Pierre Nora. Homo historicus, Paris, Perrin, 2011.
} 
Vincent Chambarlhac, « Les prémisses d'une restauration ? L'histoire enseignée saisie par le politique », Histoire@Politique. Politique, culture, société, n 16, janvier-avril 2012, www.histoire-politique.fr

profession de foi. Amour, foi, ce sont des mots que j’ai soigneusement évités, pour les remplacer par ceux qu'appelaient l'époque et le point de vue ethnologique ${ }^{32}$. »

En regard de l'histoire enseignée, le «moment Lavisse» désigne la séquence où l'histoire s'institutionnalise, dans la recherche, l'enseignement, et s'invente une première pédagogie ${ }^{33}$. Symétriquement, le «moment Decaux » serait ce moment où se ré-institutionnalise l'histoire dans son rapport à la société, autorisant une nouvelle fluidité des rapports histoire savante/ histoire enseignée, puisque la seconde réintègrerait sa mission d'originelle d'un apprentissage civique par le récit national. Il n'est donc pas dans l'emploi par Pierre Nora de «moment Decaux » de signifiant subversif, mais bien davantage l'expression d'une forme de retour à l'ordre et au genre qu'est l'histoire de France enseignée, pour peu que celle-ci ait conscience de son historicité. En ce sens, le « moment Decaux » peut se lire comme l'avers dans la fabrique scolaire de l'histoire de l'opération historiographique des Lieux de mémoire. Cette configuration intellectuelle se saisit dans l'après-coup au prix d'un oubli du grondement politique de la polémique grandement facilitée par la nouvelle « républicanisation » de l'histoire enseignée. Cette républicanisation, patente dès les programmes Chevènement de 1985, marque l'historicité de la forme républicaine en ordonnant l'étude sous la forme de nouveaux objets, dont les héritages, les symboles, les mémoires ${ }^{34}$. Nommer les origines de ce tournant comme un « moment Decaux » revient à identifier ce qui dans la figure d'Alain Decaux permet de concilier la tentation du retour à un enseignement traditionnel à la conscience même de l'historicité de celui-ci. En bref, comment le roman national peut se renouveler.

Pour Historiens et Géographes, Alain Decaux est «notre ami »; une telle amitié enjoint au journal la défense de ce dernier face aux critiques. Contre l'épithète de réactionnaire, Hubert Tison réaffirme les qualités d'homme de gauche d'Alain Decaux. À l'appui de la démonstration, ses travaux sur Blanqui l'insurgé, où il cite Maurice Dommanget et Henri Guillemin (Historiens et Géographes, ${ }^{\circ}$ 277, févriermars 1980) mais aussi les félicitations d'Albert Soboul en 1969 pour son émission sur Robespierre, ses qualités de vulgarisateur reconnues par Ernest Labrousse. Sa qualité d'académicien, longuement évoquée, s'associe dans un vibrant hommage d'Hubert Tison à son talent de « merveilleux conteur à la radio et à la télévision », gage d'《 un grand médiateur de l'histoire, souvent à l'écoute des humbles et des opprimés ». L'ensemble de ces facettes se fond dans l'engagement de « cet ardent défenseur de l'enseignement de l'histoire » digne héritier d'un grand-père instituteur (Historiens et Géographes, $\mathrm{n}^{\circ} 278$, mai 1980, p. 582-585). Ainsi ramassée, ces qualités symbolisent la possibilité d'une restauration de l'ordre de l'histoire enseignée. L'homme est de gauche, mais d'une gauche du XIXe siècle, qui disparaît en 1917 comme il le confie à Pierre Nora ${ }^{35}$. La précision mesure l'appartenance à une gauche

\footnotetext{
32 Pierre Nora, « La nation sans nationalisme », Espace-Temps, n 59-61, 1995. Repris dans Pierre Nora, Historien public, Paris, Gallimard, 2011, p. 448.

33 Patrick Garcia, J ean Leduc, «Le moment Lavisse », dans L'enseignement de l'histoire en France de l'Ancien Régime à nos jours, Paris, Armand Colin, 2003.

34 Cf. Patricia Legris, «Les programmes scolaires dans l'enseignement de l'histoire», dans Laurence de Cock (dir.), La fabrique scolaire del'histoire, Marseille, Agone, 2009, p. 28-52.

35 Pierre Nora, « Alain Decaux raconte...», op. cit.
} 
Vincent Chambarlhac, « Les prémisses d'une restauration ? L'histoire enseignée saisie par le politique », Histoire@Politique. Politique, culture, société, n 16, janvier-avril 2012, www.histoire-politique.fr

non marxiste au moment où le procès du gauchisme de Mai 68 est entendu ${ }^{36}$. En ce sens, les philippiques du Peuple français contre Alain Decaux et la pratique de l'histoire qu'il représente sont avant tout politiques, mais dans l'ordre d'une médiation par l'histoire du récit local contre le récit national. En ce sens également, la qualité d'homme d'une gauche révolue d'Alain Decaux conjure le spectre du matérialisme historique régulièrement excipé dans les années 1970 par les tenants d'une rénovation de l'enseignement d'histoire qu'ils soient dans l'institution (Suzanne Citron) ou sur ses marges (Forum-histoire notamment). Pour autant, l'essentiel politique du « moment Decaux » tient peu à la discussion - sans fin sans doute - de la définition de l'homme de gauche qu'il serait dans son rapport à l'histoire. Il porte davantage sur la symbolique de cet homme de télévision et de radio campant l'instituteur national. Dans le champ de l'histoire enseignée, la question des médias comme producteur d'histoire motive une grande part des réformes, ce jusqu'à la « réforme Haby »; elle implique systématiquement un horizon modernisateur et travaille l'essentiel des propositions de rénovation pédagogique. Dans la doxa des discours sur l'École, cette position serait réformiste, contre une position plus conservatrice assumant la chronologie, le récit national. Campant l'instituteur national, Alain Decaux reconfigure symboliquement une position où la télévision semblait, sinon l'adversaire de l'histoire enseignée, un redoutable concurrent. La figure de l'instituteur, parce qu'elle porte de premier apprentissage de l'histoire (donc de vulgarisation), et de récit convient à la cause de l'APHG. Dans cette posture d'instituteur, Alain Decaux est davantage l'homme de l'ORTF, plus que de son éclatement giscardien où l'histoire à la télévision peut davantage diviser, car mettant en scène un passé qui alors ne passe pas ${ }^{37}$. Dans le rôle d' «instituteur national » dévolu à Alain Decaux se lit l'emprise nouvelle des médias sur l'histoire. Radio ou télévision peuvent se présenter - à tort ou à raison - comme productrices d'un nouveau régime de vérité historique, ou tout du moins d'une vulgate nouvelle. Alain Decaux, instituteur national, constitue dans cette configuration le point d'équilibre idoine entre les deux pratiques du genre qu'est l'histoire de France dans son horizon civique: le cadre scolaire, le cadre médiatique. La conjonction est d'autant plus fragile qu'elle tient surtout à la mise en scène de l'émission «Alain Decaux raconte $^{38} \gg$ qui pose comme centrales la question du récit, sa dramatisation. Le présentateur se présente comme un trait d'union entre la pratique et l'enseignement de l'histoire :

«Quand j'écris [Alain Decaux] mes livres, je fais le travail classique de l'historien parce que j'ai du temps pour ma recherche. Mais pour des émissions tous les mois, ou toutes les semaines à la radio, il est bien évident que je suis obligé de plonger dans ce

\footnotetext{
36 Michaël Christofferson, Les intellectuels contre la gauche. L'idéologie antitotalitaire en France (19681981), Marseille, Agone, « Contre-feux », 2009.

37 Isabelle Veyrat-Masson, «Les guerres de mémoires à la télévision: du dévoilement à l'accompagnement », dans Isabelle Veyrat-Masson, Pacal Blanchard (dir.), Les guerres de mémoire. La France et son histoire, Paris, La Découverte, 2008.

38 L'émission est en direct, Alain Decaux dans un face à face avec la caméra. L'émission démarre en 1971, s'interrompt en 1981.
} 
Vincent Chambarlhac, « Les prémisses d'une restauration ? L'histoire enseignée saisie par le politique », Histoire@Politique. Politique, culture, société, n 16, janvier-avril 2012, www.histoire-politique.fr

qui a déjà été écrit. Est-ce si différent d'un professeur qui se trouve chargé d'un cours sur une question dont il n'ajamais exploré les sources personnellement ${ }^{39}$ ? »

On voit dans l'analogie l'implicite d'une restauration de l'édifice disciplinaire de l'histoire entre le praticien et l'enseignant.

\section{Conclure provisoirement...}

Quid du politique ? Si l'on accepte l'augure d'une restauration, la polémique - et son issue différée, mais patente dans les programmes malgré d'autres médiations politiques (échec de Savary, tournant de la rigueur) - dit que la re-fondation des rapports histoire enseignée / histoire savante sur l'idéal fantasmée de l'École de J ules Ferry (dont une certaine littérature ne cessera plus dans la décennie 1980 de proclamer la mort) implique l'irruption du tiers médiatique. La ruse de l'histoire est qu'ici cette irruption autorise une restauration, quand pour d'aucuns la prise en compte de ce nouveau producteur d'histoire pour le grand public réclamait un ajustement des pédagogies, des programmes. En ce sens, on constatera que cet épisode de l'histoire scolaire renseigne singulièrement la manière dont politiquement une époque se clôt. L'adieu aux ombres de « 68 » comme pratiques politiques d'une forme d'histoire par le bas s'énonce sous la forme d'un récit national dont s'exige la remise à l'ordre du jour. On observera alors que les problématiques des Lieux de mémoire et la polémique de 1979-1980 voisinent. Toutes deux saisissent en creux ce que l'expérience des années 1970 a défait et la nécessité d'une historicisation du genre qu'est l'histoire de France. À partir de 1985, peu ou prou, tous les programmes tiennent compte de cette historicité. La part implicite du politique dans cette dynamique tient à une réflexion dont la finalité civique tient à l'historicité du fait républicain qui donne sa place aux mémoires dans un roman national. Parce que cette place est le fruit d'un incessant compromis où l'École n'est que le cadre dans lequel s'inscrit une communication politique construite sous les auspices de la reconnaissance, le roman national est instable, par définition. « On n'enseigne plus l'histoire à vos enfants...» est un slogan alors voué à la réitération. Il implique finalement moins une pensée de l'enseignement de l'histoire et davantage une réflexion sur sa finalité civique. Politique donc. Parce qu'il est consubstantiellement lié au modèle de la IIIe République - ce jusqu'à sa lecture par les Lieux de mémoire le roman national tient à la restauration, et dans l'ordre du politique ses contempteurs sont toujours soupçonnés d'œuvrer à l'affaiblissement du lien civique.

\section{L'auteur}

Vincent Chambarlhac, maître de conférences en histoire contemporaine à l'Université de Bourgogne, Département info-com, chercheur au Centre Georges Chevrier (UMR CNRS 5605). Membre du comité de rédaction de la revue Dissidences (http://revuesshs.u-bourgogne.fr/ dissidences/). Il participe actuellement aux réflexions autour du séminaire international «Réception et production de récits de

39 Pierre Nora, « Alain Decaux raconte...», op. cit., p. 12. 
Vincent Chambarlhac, « Les prémisses d'une restauration ? L'histoire enseignée saisie par le politique », Histoire@Politique. Politique, culture, société, n 16, janvier-avril 2012, www.histoire-politique.fr

l'histoire nationale» organisé par l'UMR Éducation et politique de l'Université de Lyon II.

\section{Résumé}

La polémique de 1979 ouverte par l'Association des professeurs d'histoire et de géographie (APHG) et par Alain Decaux sur l'enseignement de l'histoire est, à bien des égards, matricielle. Une lecture politique de celle-ci questionne l'épistémologie de la discipline dans son rapport aux années 1968, dans la manière dont elle peut constituer une forme d'introduction à la nouvelle configuration historiographique qu'établissent Les Lieux de mémoire. Trois temps ordonnent la saisie de cette polémique inaugurale: le tempo du politique, le temps rétrospectif du procès des années 68, l'après-coup de l'épistémologie.

Mots clés : histoire scolaire, épistémologie, politique, récit national.

\section{Abstract}

The controversy of 1979 launched by the APHG (Association des professeurs d'histoire et de géographie) and Alain Decaux on the teaching of history is in many respects a matrix. A political interpretation of this debate questioned the epistemology of the discipline in relation to 1968. It can also be seen as anintroduction to a new configuration of the historiography, as exemplified by the famous book Les Lieux de mémoire. The tempo of the controversy is diverse: the political debate moment, the retrospective trial of the years 1968, and the long lasting epistemological effect.

Keys words : school history, epistemology, policy.

Pour citer cet article : Vincent Chambarlhac, «Les prémisses d'une restauration ? L'histoire enseignée saisie par le politique », Histoire@Politique. Politique, culture, société, $\mathrm{n}^{\circ} 16$, janvier-avril 2012, www.histoire-politique.fr 